\title{
Altered Recruitment of Motor Cortex Neuronal Activity During the Grasping Phase of Skilled Reaching in a Chronic Rat Model of Unilateral Parkinsonism
}

\author{
Borian I. Hyland, ${ }^{1}{ }^{\circledR}$ Sonja Seeger-Armbruster, ${ }^{1}$ Roseanna A. Smither, ${ }^{1,2}$ and $\mathbb{C}_{\text {Louise C. Parr-Brownlie }}^{2}$ \\ ${ }^{1}$ Department of Physiology and ${ }^{2}$ Department of Anatomy, School of Biomedical Science and Brain Health Research Centre, University of Otago, Dunedin \\ 9054, New Zealand and the Brain Research New Zealand Centre of Research Excellence
}

Parkinson's disease causes prominent difficulties in the generation and execution of voluntary limb movements, including regulation of distal muscles and coordination of proximal and distal movement components to achieve accurate grasping. Difficulties with manual dexterity have a major impact on activities of daily living. We used extracellular single neuron recordings to investigate the neural underpinnings of parkinsonian movement deficits in the motor cortex of chronic unilateral 6-hydroxydopamine lesion male rats performing a skilled reach-to-grasp task the. Both normal movements and parkinsonian deficits in this task have striking homology to human performance. In lesioned animals there were several differences in the activity of cortical neurons during reaches by the affected limb compared with control rats. These included an increase in proportions of neurons showing rate decreases, along with increased amplitude of their average rate-decrease response at specific times during the reach, suggesting a shift in the balance of net excitation and inhibition of cortical neurons; a significant increase in the duration of rate-increase responses, which could result from reduced coupling of cortical activity to specific movement components; and changes in the timing and incidence of neurons with pure rate-increase or biphasic responses, particularly at the end of reach when grasping would normally be occurring. The changes in cortical activity may account for the deficits that occur in skilled distal motor control following dopamine depletion, and highlight the need for treatment strategies targeted toward modulating cortical mechanisms for fine distal motor control in patients.

Key words: 6-OHDA lesion; extracellular recording; grasping; Parkinson's; single neuron; skilled reaching

Significance Statement

We show for the first time in a chronic lesion rat model of Parkinson's disease movement deficits that there are specific changes in motor cortex neuron activity associated with the grasping phase of a skilled motor task. Such changes provide a possible mechanism underpinning the problems with manual dexterity seen in Parkinson's patients and highlight the need for treatment strategies targeted toward distal motor control.

\section{Introduction}

Parkinson's disease is a degenerative neurological disorder that causes prominent difficulties in the generation and execution of voluntary limb movements. These deficits extend to the fine regulation of targeted skilled movements involving distal muscles, and the coordination of proximal and distal muscle activations, such as occur during reach-to-grasp movements (Benecke et al.,

Received March 28, 2019; revised Sept. 17, 2019; accepted 0ct. 16, 2019.

Author contributions: B.I.H. and L.C.P.-B. designed research; B.I.H., S.S.-A., R.A.S., and L.C.P.-B. analyzed data; B.I.H., S.S.-A., R.A.S., and L.C.P.-B. edited the paper; B.I.H. wrote the paper; S.S.-A. and R.A.S. performed research; S.S.-A. wrote the first draft of the paper.

This work was supported the Health Research Council of New Zealand Grant 12-182 to B.I.H. and L.C.P.-B.

The authors declare no competing financial interests.

Correspondence should be addressed to Brian I. Hyland at brian.hyland@otago.ac.nz.

https://doi.org/10.1523/JNEUROSCI.0720-19.2019

Copyright $\odot 2019$ the authors
1987; Castiello and Bennett, 1994; Whishaw et al., 2002; Schettino et al., 2004; Melvin et al., 2005). Similar deficits of distal components of skilled reach-to-grasp movements are seen in a chronic rat model of Parkinson's disease (Miklyaeva et al., 1994).

Determining the neural basis of such deficits is an area of active investigation. The underlying cause of the motor problems in Parkinson's disease is loss of dopamine neurons in the midbrain, whereas the executive control of normal dexterous voluntary limb movements is dependent on functioning of the cerebral motor cortex. Again, this is the case for rats (Kolb and Whishaw, 1983; Whishaw et al., 1992a; Whishaw and Coles, 1996; Whishaw, 2000), just as in primates (Brochier and Umiltà, 2007). Loss of dopaminergic projections can impinge on motor cortex function directly and indirectly. Dopamine released directly in cerebral cortex has an important role in modulating 
neurotransmission (Lewis and O'Donnell, 2000; Seamans et al., 2001a,b), and loss of this input occurs in Parkinson's disease patients (Gaspar et al., 1991) and in rats with dopamine lesions (Debeir et al., 2005; Lindenbach et al., 2015). Indirectly, loss of the large dopaminergic projections to the basal ganglia leads to disordered processing through the basal ganglia-thalamus-cortex loop, which is an important determinant of motor cortex neural activity (Albin et al., 1989; Boraud et al., 2002; Wichmann and Dostrovsky, 2011; Galvan et al., 2015). Given the convergence of direct and indirect effects of dopamine loss at the level of motor cortex, knowledge of the disordered activity of motor cortex neurons during movement is key for understanding the origin of the problems with dexterous voluntary movements seen in Parkinson's disease.

Studies of motor cortex neuronal activity in hypodopaminergic states have revealed significant abnormalities at regional and single neuron levels. At the regional level, a range of deficits including both hypo- and hyperactivation have been found in functional imaging studies of motor cortex of Parkinson's patients during movement tasks (for review, see Herz et al., 2014). Variation between studies may relate to differences in task-specific details such as whether movements involved proximal or distal joints, or were internally or externally triggered or paced, the stage of disease, or whether bradykinesia or rigidity dominate the clinical picture (Wu and Hallett, 2013; Herz et al., 2014; Planetta et al., 2014). At the single neuron level, recordings in parkinsonian primates during performance of simple, single-joint flexionextension tasks have reported a range of effects, including distorted timing, reduced amplitude of responses, reduced muscle selectivity, and decoupling of neural activity from movement parameters (Doudet et al., 1990; Watts and Mandir, 1992; Pasquereau et al., 2016). Much less is known of the single neuron correlates of deficits in distal movements and proximal-distal coordination as expressed in complex reach-to-grasp tasks. In such tasks there is remarkable homology between rats and humans in the characteristics of both normal movements and parkinsonian deficits (Whishaw et al., 1992b, 2002; Sacrey et al., 2009). In a previous rat study using an acute drug-induced model of parkinsonism, we found reduced engagement of motor cortex neurons (ParrBrownlie and Hyland, 2005). Here, we extend that work using a chronic, unilateral rat model of Parkinson's disease. The results revealed specific shifts in the balance, timing and amplitudes of firing rate modulations over the time when specific proximaldistal coordination and distal adjustments are being made for successful grasping. Such changes may account for previously reported disturbances in these movement components in chronic parkinsonism.

\section{Materials and Methods}

Animals and experiments

Adult male Wistar rats $(n=15)$ were housed in environmentally controlled conditions in a reversed $12 \mathrm{~h}$ light/dark cycle. All experiments were conducted during the rats' dark cycle and animals were restricted to $18 \mathrm{~g}$ of standard rat chow per day to ensure they were motivated to execute the reaching task to obtain palatable food. All experiments were approved by the University of Otago Animal Ethics Committee.

In overview, rats were trained to perform a skilled reaching task and the most frequently used (dominant) paw was determined. One group then underwent surgery to inject the selective neurotoxin 6-hydroxydopamine (6-OHDA) in the medial forebrain bundle of the hemisphere contralateral to the dominant paw, while another (control) group had sham surgery. Animals from the first group with successful lesion effects, and all sham lesioned animals, were then operated again, to implant chronic extracellular recording electrodes in the motor cortex of the
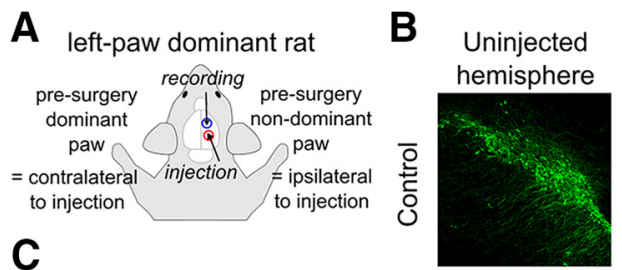

Injected hemisphere

C
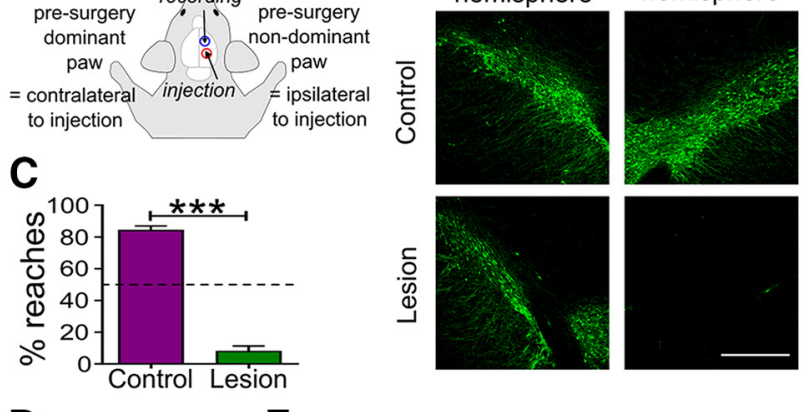

D
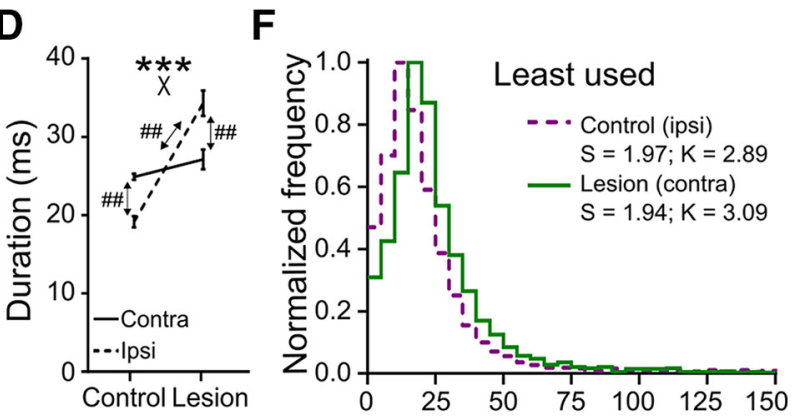

E
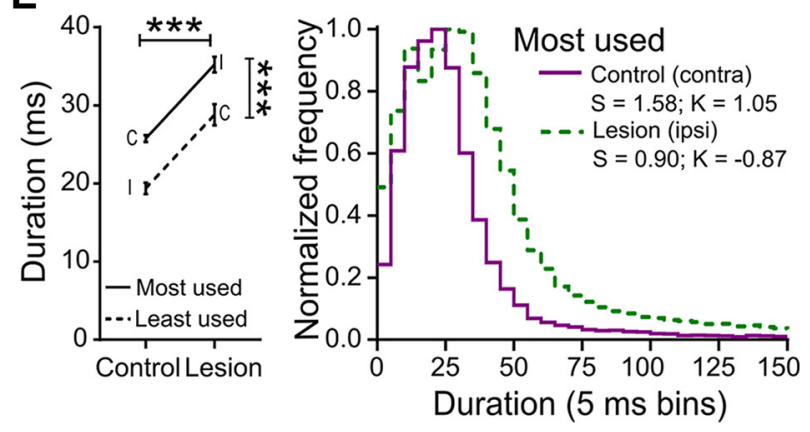

Figure 1. Histological and behavioral effects of the unilateral 6-0HDA lesion. $\boldsymbol{A}$, Schematic top view of a rat showing naming conventions. Example is for a rat in which the left paw was dominant as assessed before sham or lesion surgery. Intracerebral injections of vehicle or 6-OHDA, and recording electrode placement, were contralateral to the presurgery dominant paw, which in the remainder of the paper is referred to as the "contralateral" paw. B, Example images from coronal sections of ventral midbrain including the substantia nigra and ventral tegmental area show immunohistochemical staining for TH (green). Scale bar $0.5 \mathrm{~mm}$. C, Proportion of reaches performed postsurgery with the contralateral paw in control and lesioned animals. ${ }^{* * *} p<0.001$, Mann-Whitney $U$ test. $\boldsymbol{D}$, Mean duration of the terminal part of the reach, executed by the contralateral (Contra; solid line) and ipsilateral (Ipsi; dashed line) paws from control and lesioned rats. ${ }^{* * *} p<0.001$ for group $X$ paw interaction $(X)$, two-way ANOVA, \#\# $p<0.01$ Holm-Sidak post hoc tests. $E$, Same data as $\boldsymbol{D}$, split according to most (solid line) and least used (dashed line) paw. C, Contralateral paw; I, ipsilateral paw. ${ }^{* *} p<0.001$ for main effects of group and usage, two-way ANOVA. $\boldsymbol{F}$, Frequency distributions (peak normalized) of reaching durations (5 ms bin) for least used (top) and most used paws (bottom) in control (purple) and lesioned (green) rats. Solid lines, contralateral paw (contra); dashed lines, ipsilateral paw (ipsi). S, Skewness; K, kurtosis.

same hemisphere as the injection. In the remainder of this paper when referring to specific paws the terms "contra-" and "ipsilateral" are relative to the hemisphere recorded from (Fig. 1A).

Behavioral task and determination of presurgery dominant paw Before the first surgery, rats were trained to perform a reach-to-grasp task previously established in the laboratory (Hyland and Jordan, 1997; Hyland, 1998; Jarratt and Hyland, 1999; Parr-Brownlie and Hyland, 2005; Bosch-Bouju et al., 2014) and which has been shown to be highly homologous to human reaching, given the same starting posture, and similarly affected by parkinsonism (Whishaw et al., 1992b, 2002; Sacrey et al., 
2009; Klein et al., 2012). Briefly, rats reached through a rectangular opening in the wall of the experimental Perspex box to retrieve a cereal pellet (Coco Pops; Kellogg's) placed on a platform $30 \mathrm{~mm}$ beyond the wall. Reaches were detected by interruption of two infrared light beams positioned along the platform at 3 and $22 \mathrm{~mm}$ from the wall of the box. The latter beam was close to the food and thus marked the end of the reaching sequence and the beginning of food grasping (Hyland and Jordan, 1997). Beam interrupts were converted to transistor-transistor-logic (TTL) pulses and recorded with electrophysiological data using Spike2 software (Cambridge Electronic Design; RRID:SCR_000903).

Before sham or lesion surgery rats performed at least 4 sessions of training to establish consistent reaching behavior. The rat's dominant paw was defined as the one used for $>70 \%$ of reaches in the final training session (Parr-Brownlie and Hyland, 2005; Bosch-Bouju et al., 2014; Seeger-Armbruster et al., 2015). All rats included in this study demonstrated a paw preference in that session, producing on average $244 \pm 108$ reaches in $5 \mathrm{~min}$, with $94.4 \%$ of these executed by the dominant paw.

\section{Surgical procedures}

Dopaminergic lesions. Unilateral dopaminergic pathway lesions were performed under aseptic conditions and antibiotic cover (amphoprim, 30 $\mathrm{mg} / \mathrm{kg}$; Virbac Animal Health) as described previously (Bosch-Bouju et al., 2014). Rats weighing $337 \pm 15$ g were deeply anesthetized using ketamine/medetomidine/atropine $(75 / 0.5 / 0.06 \mathrm{mg} / \mathrm{kg}$, s.c., Phoenix Pharm/Pfizer). Desmethylimipramine (15 mg/kg, i.p., Sigma-Aldrich) was injected $30 \mathrm{~min}$ before the neurotoxic lesion to prevent degeneration of noradrenergic neurons. Rats were placed in a stereotaxic frame (flat skull position) and injected s.c. along the intended incision line with the long-acting local anesthetic lopaine (2\%; Ethical Agents). The skull was exposed by a midline sagittal incision and a hole was drilled at the coordinates for the medial forebrain bundle at anteroposterior (AP) +4.40 $\mathrm{mm}$ and mediolateral (ML) $1.20 \mathrm{~mm}$ (relative to lambda) according to standard atlas coordinates (Paxinos and Watson, 2007). The neurotoxin 6-OHDA ( $6 \mu \mathrm{g}$ in $3 \mu \mathrm{l} 0.01 \%$ ascorbic acid in $0.9 \%$ saline solution; Sigma-Aldrich) was infused in the medial forebrain bundle $(-8.30 \mathrm{~mm}$ below skull surface) via a 26-gauge cannula at $1 \mu \mathrm{l} / \mathrm{min}$ to induce the unilateral dopaminergic lesion. Control rats were sham lesioned with an equivalent volume of the vehicle alone. The cannula remained at the target site for $5 \mathrm{~min}$ after the infusion stopped before being slowly withdrawn to prevent diffusion of the neurotoxin along the injection track. The incision was sutured, the rat was injected s.c. with the long-acting analgesic carprofen $(5 \mathrm{mg} / \mathrm{kg}$, Carprieve; Norbrook) and $5 \mathrm{ml}$ of $0.9 \%$ saline, removed from the stereotaxic frame and then anesthesia was reversed with Antisedan (atipamezole $2.5 \mathrm{mg} / \mathrm{kg}$, s.c., Pfizer). Twice daily postoperative checks were performed for 1 week.

To select successfully lesioned rats to undergo subsequent implantation of recording electrodes, 4-5 d after surgery we quantified paw use with the step test (Olsson et al., 1995). In this test the ratio of steps performed with each paw is normally $\approx 1.0$. Only rats with a ratio (contralateral paw/ipsilateral paw) of $\leq 0.1$ were taken further in the study. In these selected rats $(n=7)$ the mean $( \pm \mathrm{SD})$ ratio was $0.06 \pm 0.04$, whereas in sham-lesioned control rats it was $0.95 \pm 0.14(n=8)$.

Electrode implantation. Extracellular recording electrodes were constructed from a bundle of 8 wires (0.0007-inch nichrome covered in formvar; A-M systems). One end of each wire was stripped of the formvar covering and soldered into a gold Amphenol pin (Connector Systems), which was then inserted into a nine-way mini-connector (Science Technology Centre, Carleton University). One end of a tinned copper wire $(0.152 \mathrm{~mm}$; RS Components) was soldered into a gold Amphenol pin and inserted into the mini-connector, and the other end was soldered to a stainless-steel screw serving as the earth screw (Bosch-Bouju et al., 2014). The wire bundle was inserted into a stainless-steel cannula and glued in place with cyanoacrylate (Supaglue; Selleys Chemical). The tip of each wire was trimmed with a new scalpel blade. Electrodes were electroplated to lower the impedance using a $-2 \mu \mathrm{A}$ current for $5 \mathrm{~s}$ in a gold-plating solution (5355; SIFCO Selective Plating) containing multiwalled carbon nanotubes ( $<8 \mathrm{~nm}$ diameter; Cheap Tubes) (Ferguson et al., 2009). Electrode impedances after gold-plating were between 0.1 and $0.9 \mathrm{M} \Omega$. The electrode assembly was fixed on a microdrive to allow vertical advancement through the motor cortex in $\sim 46 \mu \mathrm{m}$ steps.

Two weeks after the 6-OHDA or sham lesion, rats underwent a second surgery to implant recording electrodes in the motor cortex of the same hemisphere (Fig. 1A). The surgical procedures were identical to the dopaminergic lesion, except that holes were drilled in the skull over the motor cortex (AP $+1.00 \mathrm{~mm}$, ML 2.25 or $2.40 \mathrm{~mm}$, relative to bregma) targeting M1 as defined by Paxinos and Watson (2007). To complete implantation, the electrode was lowered so that all wires were in the brain, resulting in initial implantation depths for individual wires of $0.64-1.30 \mathrm{~mm}$ below the dura. The earth screw for recordings was positioned in the skull above the occipital lobe in the other hemisphere and five additional stainless-steel screws were implanted into the skull to secure the microdrive to the rat's head using dental acrylic (Vertex Dental; AA Zeist). A mini-connector and protective cover with holes to access the microdrive were fixed on the rat's skull using dental acrylic.

\section{Single-unit recordings}

Recording experiments began 6-7 weeks after the electrodes were implanted, which correspond to 8-9 weeks after the lesion surgery. Rats were placed in the reaching box and connected to the recording cable which was attached to an electrical rotary joint (Doric Lenses). Action potentials were bandpass filtered $(0.3-6 \mathrm{kHz})$ and amplified $(2000 \times)$ using a CyberAmp signal conditioner (Molecular Devices), with the earth serving as the reference signal for all wires. Channels showing distinguishable single-cell activity were recorded at $37 \mathrm{kHz}$ sample rats using a Power 1401 A-D converter and Spike2 software (Cambridge Electronic Design) that digitized the data and stored spike waveforms and timestamps. Action potentials from individual neurons were initially discriminated from each other and noise using the template matching spike sorting algorithm in Spike2. Clusters were refined by subsequent principal component analysis if necessary. Sessions were video recorded using the Spike2video feature, which synchronizes the video frames to the electrophysiological recordings.

\section{Experimental design and statistical analysis}

We analyzed motor cortex neuronal activity from freely moving control $(n=8)$ and 6-OHDA lesioned $(n=7)$ rats. Two separate epochs of 10 min each were recorded during each session, in randomized order. In one, the "task" epoch, cereal pellets were provided at the reaching platform for the rat to retrieve. Rats were continually observed and manual keystrokes used to mark on the data file the reaches executed with the contralateral paw, so that we could analyze motor cortex neuronal activity associated with those reaches. As previously described (Bosch-Bouju et al., 2014), the pellet was manually restrained until animals made a reach with the contralateral, affected paw, to encourage continued use of that paw in lesioned rats. We quantified the number of reaches and the duration of the terminal part of the reach as defined by the two lightbeam interrupts. In the other, "no-task" epoch, no pellets were provided and rats expressed a range of natural behaviors, including quiet rest, grooming, etc. For analysis of activity in the no-task epoch, periods of quiet rest were identified off-line from the Spike2 video.

For each discriminated neuron, we measured the average action potential waveform width $(\mathrm{ms})$ and amplitude $(\mu \mathrm{V})$. Overall characterization of recorded neurons for the purpose of determining if there were specific subpopulations based on waveform characteristics or firing rate was performed by calculating average firing rates and spike waveform peak-to-trough width and amplitude using all spikes recorded across both task epochs (total $20 \mathrm{~min}$ ).

For analysis of the effect of 6-OHDA lesion on basic neuron firing characteristics (overall rate and bursting measures), we separately analyzed the task and no-task epochs. To characterize overall average activity during reaching, we analyzed the second $30 \mathrm{~s}$ period of reaching during the task epoch, at which time rats had established repetitive reaching behavior, while for "resting" activity we used a period of $30 \mathrm{~s}$ of continuous quiet rest from the no-task epoch, determined from video recordings. Spike train pattern was characterized by measures of regularity and bursting. Regularity was quantified by the interspike interval coefficient of variation (ISI CV), obtained by dividing the SD of all ISI in each $30 \mathrm{~s}$ 
epoch by the mean ISI. Spike trains were classified as "bursting" or "nonbursting" using the density discharge histogram method, as described previously (Kaneoke and Vitek, 1996; Parr-Brownlie et al., 2007; BoschBouju et al., 2014). For this analysis, a burstiness index of 0.5 was used so that the bin width of the density discharge histogram was twice the mean ISI for each recording. A spike train was classified as bursting if it met the following criteria: there were $\geq 3$ spikes in the bursts, the distribution of the discharge density histogram was significantly different from a Poisson distribution ( $\chi^{2}$ test set at a significance level of 0.01$)$, the histogram was positively skewed, the firing rate was $\geq 0.5$ spikes s $^{-1}$, and had $>5$ bursts per 1000 spikes (Parr-Brownlie et al., 2007, 2009; Walters et al., 2007; Bosch-Bouju et al., 2014). The bursting intensity of these neurons was quantified by calculating bursts/100 spikes, mean spikes/burst, and percentage spikes in bursts.

To detect and quantify reach-related modulations in firing rate of individual neurons we calculated perievent time histograms (PETH; bin width $20 \mathrm{~ms}$ ) averaged from reaches performed by the contralateral paw in the session. Time 0 on the PETH was set at the moment the paw broke the infrared beam closest to the food, marking the end of the reaching sequence and the beginning of food grasping (Whishaw and Pellis, 1990; Whishaw et al., 1992b; Whishaw and Gorny, 1994; Hyland and Jordan, 1997). Only recording sessions in which at least 10 individual reaches contributed to the PETH were analyzed. PETH bin counts were converted to instantaneous frequency to normalize for different trial numbers between experiments. The baseline firing rate for each PETH was calculated as the average spikes $\mathrm{s}^{-1}$ during the period -1.0 to $-0.5 \mathrm{~s}$ before the light beam crossing, corresponding to the period immediately before earliest reach-related muscle activations (Hyland and Jordan, 1997). During this period rats establish a relatively consistent posture in preparation for the reach (Whishaw and Pellis, 1990), thus providing a uniform behavioral baseline across trials (Parr-Brownlie and Hyland, 2005). Rate-increase and rate-decrease modulations were defined as peaks or troughs of $\geq 2$ consecutive bins with firing rates that exceeded $\pm 2 \mathrm{SD}$ of the baseline rate. The modulation duration was determined from the first to the last consecutive bins that exceeded this threshold. Some neurons had a floor effect in the rate-decrease (trough) response, with very low baseline firing rates in the PETH resulting in negative numbers when subtracting 2 SD. For these neurons, we applied an additional criterion for rate-decrease responses, defined by Galvan et al. (2016). Here, periods with zero bin counts lasting at least 2 bins longer than the longest silent segment during the PETH baseline were also defined as a rate-decrease modulation. Latencies were measured as the time from reach end at time 0 until the first bin meeting specified significance criteria for rate-increase or decrease responses.

To generate population grand-average PETH, individual cell PETH firing rates were first $z$-score normalized to take into account variation in baseline firing rates between cells and between groups of rats. $z$-scores were calculated for each bin $\left(Z_{\mathrm{i}}\right)$ relative to the baseline mean firing rate as $Z_{\mathrm{i}}=\left(F_{\mathrm{i}}-F_{\mathrm{B}}\right) / \mathrm{SD}_{\mathrm{B}}$, where $F_{i}$ was the instantaneous frequency in the bin and $F_{\mathrm{B}}$ and $\mathrm{SD}_{\mathrm{B}}$ the baseline mean and $\mathrm{SD}$, respectively. For quantitative analysis, we divided the grand average PETH into 6 subperiods relative to time 0 : $\# 1$ from -360 to $-240 \mathrm{~ms}$, $\# 2$ from -240 to $-180 \mathrm{~ms}$; $\# 3$ from -180 to $-40 \mathrm{~ms}$; 4 from -40 to $+40 \mathrm{~ms}$; $\# 5$ from +40 to +180 $\mathrm{ms}$; and \#6 from +360 to +500 ms. Periods \#1, 2, 34 and 5 approximately correspond to the locate, lift/aim, advancement, pronate/grasp, and retraction phases of the reach-to-grasp movement, respectively, each phase having specific associated muscle activations and deactivations (Whishaw and Pellis, 1990; Whishaw et al., 1992b; Hyland and Jordan, 1997). Period \#6 could include movements associated with bringing food to the mouth, consumption and/or postural adjustments to prepare for the next reach. For statistical comparisons we calculated the net area under the normalized PETH curves for each period by summing positive and negative $z$-score bin values, as described in Bosch-Bouju et al. (2014).

Statistical analyses were performed using Prism 8.0 (GraphPad Software; RRID:SCR_002798). Analysis of numerical measures were made with Student's $t$ or Mann-Whitney U tests or two-way repeated measures or mixed-effects ANOVA followed by Holm-Sidak post hoc tests, as appropriate. Analysis of ratios, percentages, or proportions was made with
Mann-Whitney $U$, Fisher's exact, or $\chi^{2}$ tests. Values in text report mean $\pm \mathrm{SD}$; data are shown as mean \pm SEM in figures.

\section{Histology}

At the end of experiments, rats were anesthetized with pentobarbital (100 $\mathrm{mg} / \mathrm{kg}$, i.p.; Provet). The last recording sites were marked by passing a $+20 \mu \mathrm{A}$ current for $30 \mathrm{~s}$ (A365 Stimulus Isolator, World Precision Instruments) through two of the wires with the largest number of recorded neurons. Rats were transcardially perfused using $10 \%$ sucrose solution followed by $4 \%$ paraformaldehyde (PFA) in $0.1 \mathrm{M}$ phosphate buffer at $\mathrm{pH}$ 7.4. Brains were extracted from the skull $\sim 4 \mathrm{~h}$ after the perfusion, postfixed in 4\% PFA overnight to maximize fixation and then immersed in $30 \%$ sucrose for at least $48 \mathrm{~h}$. Coronal sections $(40 \mu \mathrm{m})$ were cut on a freezing microtome (Leica) and stored at $-20^{\circ} \mathrm{C}$ in cryoprotectant. Successful 6-OHDA lesion was confirmed on midbrain slices containing the substantia nigra pars compacta (SNc) and ventral tegmental area (VTA) using a tyrosine hydroxylase (TH) antibody (1/300, rabbit polyclonal anti-TH, AB152, Abacus, RRID:AB_390204; overnight at $4^{\circ} \mathrm{C}$ ) tagged with a fluorophore (1/1000, goat anti-rabbit IgG Vector Dylight 488 , In vitro technologies, DI-1488, RRID:AB_2336402; $4 \mathrm{~h}$ at room temperature) or chromogen (DAB substrate kit, In Vitro Technologies, SK-4100, RRID:AB_2336382) revealing steps. Tissue was mounted on subbed slides. For fluorophore tagged sections progold anti-fade mountant with DAPI (Life technologies, P36931) was applied and tissue was visualized on the Nikon $1 \mathrm{AR}$ confocal microscope using $4 \times, 10 \times$, and $40 \times$ objectives. Neutral red (FD Technologies) staining was used to confirm the placement of the electrode in motor cortex sections containing the track and electrocoagulation marks. Neutral red and chromogen labeled tissue were visualized on a light microscope (Olympus CX21LED) using $4 \times$ and $10 \times$ objectives.

\section{Results}

\section{Effects of 6-OHDA lesion on reaching behavior}

As shown in Figure $1 B$, immunohistochemical staining of midbrain sections for $\mathrm{TH}$ confirmed almost complete loss of dopamine neurons in the ipsilateral SNc and VTA in the 6-OHDA lesioned rats. As expected, the lesion had a dramatic effect on paw usage in the reaching task, causing a profound reduction in the number of reaches performed by the previously dominant, contralateral paw, averaged over all recording sessions from the 3-10 months experimental period following surgery. Whereas control rats continued to predominantly use this paw, lesioned rats performed $<10 \%$ of reaches with it (Fig. $1 C$; $p<0.001$, MannWhitney $U$ test). Instead, lesioned rats switched to using the forelimb ipsilateral to the lesion, as previously described in unilateral rat models of chronic parkinsonism (reviewed by Miklyaeva et al. (1994)). With this strategy they achieved an average total number of reaches per $5 \mathrm{~min}$ in each recording session of $393 \pm 47$ ( $n=134$ sessions), which was not significantly different to control rats ( $493 \pm 215$ reaches, $n=96$ sessions; $p=0.232$, two-tailed Mann-Whitney $U$ test).

However, analysis of the duration (reflecting the average velocity) of the terminal part of the reaches found no significant slowing of those reaches that were performed with the contralateral paw in lesioned animals. While bradykinesia of movements in general is a major feature of parkinsonism, the lack of slowing is consistent with previous results using the same task (BoschBouju et al., 2014), and may relate to the fact that the quantified interval includes the deceleration phase of the reach, when velocities in controls and parkinsonian rats are known to converge (Whishaw et al., 1994). Another possibility is that the expressed velocity of this part of the reach in lesioned animals is a balance of two opposite influences deriving from the lesion that cancel each other out; slowing, due to the effect of dopamine lesion, counteracted by speeding, due to this limb no longer being appropriately controlled to optimize grasping success. 
The latter effect is supported to some extent by consideration of velocities achieved by the ipsilateral paw. The two-way analysis revealed a complex effect, shown in Figure $1 D$, with a significant paw (ipsilateral, contralateral) $\times$ group (lesion, control) interaction $\left(F_{(1,265)}=50.23, p<0.001\right.$, two-way ANOVA). Unpacking this interaction with post hoc tests confirmed that this was due to a different effect of the lesion on the two paws. Specifically, while there was no significant difference in duration between control and lesioned animals for the contralateral paw, reaches by the ipsilateral paw were significantly longer in duration in lesioned animals than in control animals. In lesioned rats the typical duration for the ipsilateral paw was $\sim 34 \mathrm{~ms}$ (average velocity $\approx 0.6$ $\left.\mathrm{m} \mathrm{s}^{-1}\right)$, compared with $\approx 19 \mathrm{~ms}\left(\approx 1 \mathrm{~m} \mathrm{~s}^{-1}\right)$ in controls $(p<$ 0.01 , Holm-Sidak's multiple-comparisons test). In addition, the within-group post hoc contrasts revealed that in control animals reach duration for the contralateral paw was significantly longer than for the ipsilateral paw $(p<0.01)$. In lesioned animals, the opposite was true, with reaches by the ipsilateral paw having longer durations than reaches by the contralateral paw $(p<$ 0.01 ). These results are the reverse of what might have been intuitively expected, given that in control animals the contralateral paw is the dominant one, and in lesioned animals the contralateral paw is the one expected to be affected by the lesion.

We hypothesized that factors related to paw use strategy may contribute to these unexpected findings. We therefore reanalyzed the effect of the lesion on reach duration using levels for the paw factor defined by postsurgery use (most used, least used) rather than by laterality relative to the lesion. As described above, in control animals the contralateral paw remained the most used after the surgery, whereas in lesioned animals, the contralateral paw became the least used. The outcome of this reanalysis is shown in the interaction plot in Figure $1 E$, which is the same data as in Figure $1 D$ but split by postsurgery paw usage. There was a clear and consistent main effect of paw usage $\left(F_{(1,412)}=43.15\right.$, $p<0.001)$ with the most used paw having the longer duration in both groups. Defining paw by usage in this analysis also brought out a main effect of group; overall, reaches had longer duration (slowed) in lesioned animals $\left(F_{(1,412)}=93.71, p<0.001\right)$. It is important to reiterate however that there was no difference between groups for reaches by contralateral paws (the reaches used for subsequent analysis of neural activity), as described above. Figure $1 F$ shows frequency distributions of reaching durations to illustrate how reaching performance changed across group and paw according to usage. The distributions for least used paws (top panel) were significantly different from for most used paws (bottom) for both control and lesioned animals $(p<0.001$ for both comparisons, $d=0.62,0.33$ respectively, KolmogorovSmirnov test), being narrower and left shifted with higher skewness and kurtosis values for the distributions for the least used paws relative to the other paw within each group. These analyses are consistent with movement by the least used paw being more ballistic over the terminal phase than for the most-used paw, in both lesioned and control animals.

Slower movement by the most-used paw in both groups of rats may reflect a control strategy optimized for goal achievement, given that during the phase of the reach quantified here accurately coordinated proximal and distal movements are occurring that are crucial for successful grasping of food (Whishaw and Pellis, 1990; Whishaw et al., 1992b; Whishaw and Gorny, 1994). That this slowness of the most-used paw was particularly exaggerated in the lesioned animals may reflect an adaptive control strategy rather than abnormality of limb control (Latash and Anson, 1996). For instance, before surgery, this paw was the non- dominant, and thus least practiced one, and in addition postural support mechanisms are impaired following unilateral lesions (Miklyaeva et al., 1994); additional slowing may help compensate for these factors to improve reach success. In addition, more direct effects of the lesion on control of this paw may contribute. Sufficiently large "unilateral" lesions can affect ipsilateral limb control, through loss of crossed dopamine axons and impaired activity in ipsilateral corticospinal projections (Vergara-Aragon et al., 2003). Consistent with this we did not see such extra slowing in a previous study where there was less pronounced akinesia, suggesting a smaller dopamine lesion (Bosch-Bouju et al., 2014). Because recordings were limited to one hemisphere, we were not able to investigate neural correlates of the properties of reaching by this limb in lesioned animals.

\section{Neuronal recording database}

A total of 80 neurons were recorded during reaching task performance in control rats and 102 in 6-OHDA lesioned rats. Electrode tracks were located within the motor cortex regions M1 and M2 as defined by Paxinos and Watson (2007) between AP + $0.48-$ +3.00 , ML $2.00-2.50$ relative to bregma (Fig. $2 A$ ), but almost all neurons $(179 / 182,98.4 \%)$ were recorded from tracks in M1.

For initial characterization of basic neuronal electrophysiological properties we pooled data across both no-task and reachtask epochs. All recorded neurons had biphasic negative-positive extracellular action potential waveforms, and, as illustrated by the examples in Figure $2 B$, included both narrow and broad waveforms as defined by trough-peak duration. The 3-D scatter plot in Figure $2 C$ shows action potential waveform duration, amplitude and mean firing rate for all recorded cells in control rats, with the equivalent data for lesioned animals in Figure $2 D$. These plots revealed a clear gap in the distribution of AP durations at $0.3-0.4 \mathrm{~ms}$ in both groups. We therefore categorized broad and narrow waveforms as being greater or $<0.4 \mathrm{~ms}$ in duration, respectively.

The majority of action potentials in both control and 6-OHDA lesioned animals (71/80, 88.8\% and 98/102, 96.1\%, respectively) were broad, with only 13 neurons meeting criteria for narrow waveforms across both groups of rats $(7.1 \%$ overall). The mean duration for broad action potentials was $\approx 0.52 \mathrm{~ms}$ and for narrow $\approx 0.25 \mathrm{~ms}$, and these durations were the same in both groups of rats (broad: control $=0.52 \pm 0.05$, lesioned $=0.53 \pm$ $0.05, t_{(167)}=1.491, p=0.138$; narrow: control $=0.25 \pm 0.03 \mathrm{~ms}$, lesioned $=0.25 \pm 0.02 \mathrm{~ms}, \mathrm{t}_{11}=0.196, p=0.848$, unpaired $t$ tests). These values are consistent with previous reports of broad and narrow waveforms in rat motor cortex (Barthó et al., 2004; Parr-Brownlie and Hyland, 2005) and primate cortex (Wilson et al., 1994), which are thought to represent pyramidal neurons and interneurons, respectively (Barthó et al., 2004). The waveform amplitudes of narrow spikes were also at the lower end of the range of amplitudes overall, as previously described in both primates and rats (Wilson et al., 1994; Parr-Brownlie and Hyland, 2005). However, with the numbers available their mean amplitude was not significantly different to that of broad spikes, in either control (broad $101 \pm 32 \mu \mathrm{V}$, narrow $85 \pm 9 \mu \mathrm{V} ; t_{(78)}=$ 1.483, $p=0.142$, unpaired $t$ test) or lesioned animals (broad $113 \pm 42 \mu \mathrm{V}$, narrow $83 \pm 12 \mu \mathrm{V} ; t_{(100)}=1.414, p=0.160$, unpaired $t$ test). There were also no differences in mean firing rate between broad and narrow spiking cells, either in control rats (broad $7.6 \pm 7.8$ spikes s $^{-1}$, narrow $4.0 \pm 4.7$ spikes s $^{-1} ; t_{(78)}=$ $1.348, p=0.182$, unpaired $t$ test) or in lesioned animals (broad

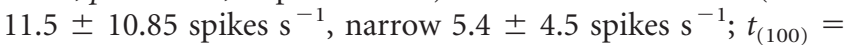
$1.115, p=0.267$, unpaired $t$ test). This is consistent with previous 
A
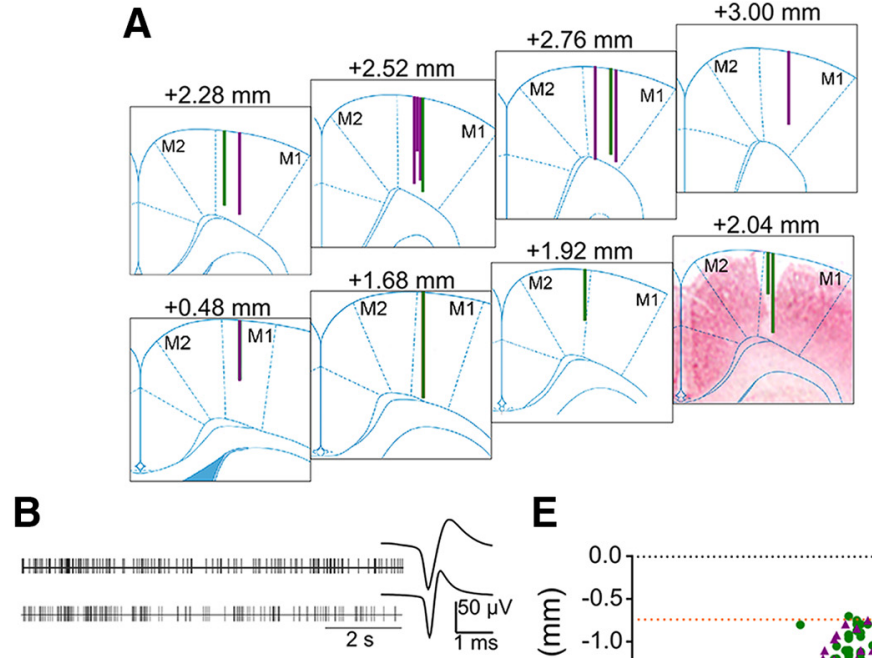

C Control

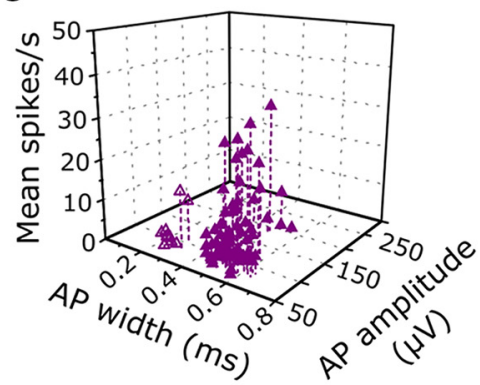

D Lesion

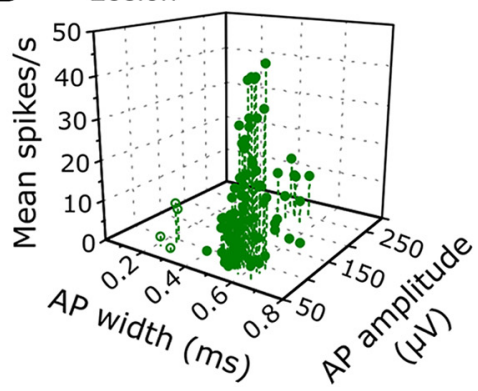

E
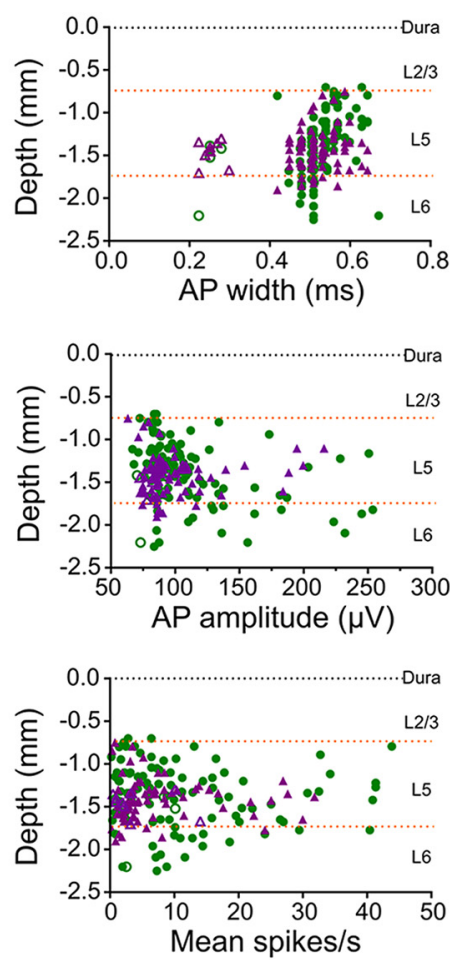

Figure 2. Characteristics of neuronal recordings. $\boldsymbol{A}$, Lines on atlas sections at indicated anteroposterior positions relative to bregma show positions of recording tracks in control (purple) and 6-OHDA lesioned rats (green). Section at $+2.04 \mathrm{~mm}$ is merged with a neutral-red stained coronal section from the equivalent histological section for the track which penetrated deeper into cortex. M1, M2: subregions of motor cortex as defined in atlas of Paxinos and Watson (2007). $\boldsymbol{B}$, Representative spike train activity and average action potential waveforms of a broad (top) and narrow spike neuron (bottom). C, Points in scatter plot show the relationship between mean firing rate, action potential (AP) width and AP amplitude for each neuron in control rats. Narrow and broad waveforms are indicated by open and filled symbols, respectively. $\boldsymbol{D}$, As for $\boldsymbol{C}$, for neurons from lesioned rats. $\boldsymbol{E}$, Scatter plots show recording depth of each neuron against AP width (top), AP amplitude (middle), and mean firing rate (bottom). Purple triangles and green circles indicate neurons from control and lesioned animals, respectively. Dashed horizontal lines indicate the approximate depth of borders between cortical layers $2 / 3,5$, and 6 .

findings in rats (Parr-Brownlie and Hyland, 2005) but contrasts with primates, in which the average firing rate of motor cortex neurons with narrow and small amplitude spike waveforms (presumed to be interneurons) is higher than for neurons with broad large amplitude waveforms, presumed to be projection neurons (Wilson et al., 1994).

Among the broad action potential neurons, the displays in Figure 2, $C$ and $D$, hint at the possibility of further subdivisions, such as those with very high firing rates or very large amplitudes. However, these divisions were not clearly demarcated using these electrophysiological criteria alone. To determine if the cortical layer that cells belonged to may account for some of the variation in the electrophysiological properties given different cellular morphologies and input-output connectivity, we constructed scatter plots of firing rate, action potential width and amplitude against recording depth, shown in Figure 2E. The plot of action potential width against depth (Fig. 2E, top) confirmed the clear break between narrow waveform neurons and the rest of the population, and furthermore revealed that these neurons were also highly clustered by depth, with all but one occurring over a depth range calculated to be in deep layer 5 . However, the scatter plots for action potential amplitude (Fig. 2E, middle) and firing rate (Fig. $2 E$, bottom) did not indicate depth specific clustering that would justify separating the broad waveform neurons into subgroups. Of the 13 narrow waveform neurons, only four were recorded in lesioned rats, so quantitative analysis of the effect of 6-OHDA lesion on the properties of this class was not possible. All subsequent descriptions and analyses therefore refer only to the broad waveform neurons. In sham lesioned animals, six rats yielded four to nine broad-spike neurons each, one yielded 15, and 17 neurons were recorded from one rat. In 6-OHDA lesioned animals, two rats yielded three and four neurons, four yielded 14-16 neurons each, and one rat provided 30 neurons.

\section{Effect of 6-OHDA lesion on overall firing properties}

To examine the effect of 6-OHDA lesion on overall firing rate and pattern we analyzed each epoch (task and no-task) separately. During the task epoch rats spent most of the time performing a highly repetitive reaching task for reward, whereas during the no-task epoch animals engaged in a range of spontaneous behaviors. For analysis of resting activity we therefore restricted analysis to periods of $30 \mathrm{~s}$ of continuous quiet rest in each notask epoch, determined from video recordings, and compared data from this epoch with the second $30 \mathrm{~s}$ period of the reaching task block, at which time rats had established repetitive reaching behavior. Because not all recordings included $30 \mathrm{~s}$ of contiguous quiet rest the number of cells available for this analysis (controls $n=30$ neurons in 6 rats, lesioned $n=45$ neurons in 7 rats) was less than the total recorded. Figure $3 A$ shows the result of the analysis of average firing rate. This revealed a significant epoch $\times$ Group interaction $\left(F_{(1,11)}=\right.$ $6.45, p=0.028$, repeated-measures ANOVA). As suggested from inspection of the interaction plot, post hoc contrasts across epochs within each group confirmed that the interaction was due to firing rates being higher during reaching than during rest in lesioned rats $(p=0.013$, Holm-Sidak's multiple-comparisons test) but not in control rats $(p=0.731)$. Further studies are 

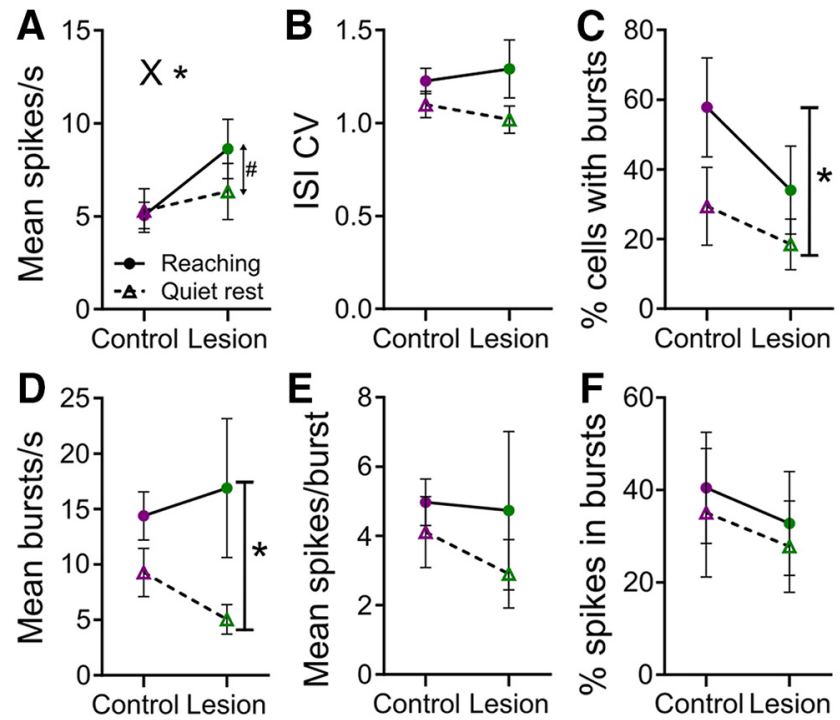

Figure 3. Effect of 6-OHDA lesion on neuronal activity measures averaged over $30 \mathrm{~s}$ of recording during task and no-task epochs. $A$, Mean firing rate in task (filled circle and solid line) and no-task epochs (open triangle and dashed line), in control (purple) and lesioned rats (green). $X^{*}$ indicates $p<0.05$, for interaction (ANOVA); $\# p<0.05$ for Holm-Sidak's post hoc contrast for rest vs reaching in lesioned rats. $\boldsymbol{B}$, Interspike interval coefficient of variation (ISI CV). C, Percentage of neurons classifed as bursting. ${ }^{*} p<0.01$, main effect of epoch (ANOVA). $\boldsymbol{D}$, Mean number of bursts per second in neurons defined as being bursty. ${ }^{*} p<0.05$, main effect of epoch (mixed-effects model). $\boldsymbol{E}$, Mean number of spikes per burst in bursting cells. $\boldsymbol{F}$, Mean percentage spikes in bursts in bursting cells.

required to explore this isolated finding. Dopamine inputs to motor cortex mediates cortical plasticity for motor learning (Hosp et al., 2011), and reward cues may increase motor cortex excitability (Thabit et al., 2011), but how reduced dopaminergic innervation in lesioned animals would lead to increased firing rate during task execution remains unclear.

Importantly for the present study, the direct between-group post hoc contrasts revealed no significant difference between lesioned and control rats, during either quiet rest $(p=0.594)$ or reaching $(p=0.142)$. While periods during the reaching epoch were specifically selected during which reaching was being performed there was still a chance rats may have paused between some reaches and performed other movements. Therefore we also analyzed the baseline period for each PETH recorded during the task epoch. This period immediately preceded the reach, when rats adopt stereotyped preparatory postures (Whishaw and Pellis, 1990; Whishaw et al., 1992b), and so the behavioral state would likely be relatively consistent. However this contrast was also not significant, although there may have been a trend for the average rate during this period to be higher in lesioned animals $\left(9.3 \pm 8.6\right.$ spikes s $\left.^{-1}\right)$ than in control rats $\left(7.3 \pm 3.4\right.$ spikes s $^{-1}$; $t_{(13)}=0.622, p=0.0545$, unpaired $t$ test $)$.

Previous findings on the effect of reduced dopamine function on overall motor cortex neuron firing rate in awake rats have been mixed, including no change, as here (Costa et al., 2006), only transient changes (Dejean et al., 2012), or a slight reduction (Parr-Brownlie and Hyland, 2005), and one in anesthetized rats finding an increase (Degos et al., 2013). Such mixed results may reflect sampling different neuronal phenotypes (Pasquereau and Turner, 2011, 2013; Pasquereau et al., 2016), and support the conclusion that simple changes in basal firing rate in basal ganglia neurons are unlikely to alone account for the deficits in movement performance observed in Parkinson's disease models (Boraud et al., 2002).
Analyses of firing pattern parameters are shown in Figure $3 B-F$. For ISI CV, a general measure of regularity of firing (Fig. $3 B)$, there was no significant effect of either epoch $\left(F_{(1,11)}=2.66\right.$, $p=0.13$, ANOVA $)$ or group $\left(F_{(1,11)}=0.01, p=0.921\right)$ and no interaction $\left(F_{(1,11)}=0.35, p=0.57\right)$. However, the percentage of cells in each rat that were classified as having bursting activity (Fig. 3C) was significantly higher during reaching than during rest (main effect of epoch, $F_{(1,11)}=5.23, p=0.043$ ). This may be due to phasic reach-related activity in cells that did not spontaneously burst during quiet rest, but other global effects such as the presence of reward cannot be ruled out (e.g., Thabit et al., 2011). There was no effect of the lesion (group, $F_{(1,11)}=1.76, p=$ 0.21 , and no interaction $\left(F_{(1,11)}=0.44, p=0.52\right)$. For analysis of bursting rate (burst episodes per second, Fig. $3 D$ ) and number of spikes in bursts (Fig. $3 E$ ) only cells that were defined as bursting were included, and some individual rats did not have bursting cells in one or other behavioral condition, so $n$ was different across behavioral epochs $(n=4$ rats in both control and lesion groups for no-task epoch, and 5 rats in both groups for the task epoch). Therefore mixed-model analysis was used to allow missing data values. The bursting rate in bursting cells was higher during reaching than rest (main effect of epoch, $F_{(1,6)}=6.58, p=$ 0.043 , mixed-effects model), again possibly due to the existence of reach-related modulations or modulation by reward. There was no effect of the lesion (group, $F_{(1,8)}=0.059, p=0.81$ ) and no interaction $\left(F_{(1,6)}=0.197, p=0.67\right)$. Finally, there were no significant main effects of group or epoch, or interactions, for the mean number of spikes in each burst event (Fig. 3E; group: $F_{(1,8)}=$ $0.26, p=0.88$; epoch: $F_{(1,6)}=0.496, p=0.51$; interaction: $F_{(1,6)}=$ $0.70, p=0.43$; mixed-effects model), or the overall percentage spikes in bursts (Fig. $3 F$; group: $F_{(1,14)}=0.10, p=0.75$; epoch: $F_{(1,14)}=0.11, p=0.75$; interaction: $F_{(1,14)}=0.00, p=0.95$; mixed effects model). These results contrast with increased bursting reported in several (Goldberg et al., 2002; Pasquereau and Turner, 2011; Degos et al., 2013) but not all (Parr-Brownlie and Hyland, 2005) studies. Again, this may depend on the sample of neurons (Pasquereau and Turner, 2011), or differences in the expression of symptoms between parkinsonian models; for instance, limb tremor is not a strong feature in rodent models (Bosch-Bouju et al., 2014). Lack of change at the cortical level in bursting may also reflect that bursting activity seen in parkinsonian basal ganglia is not strongly reflected in motor thalamus neurons (Pessiglione et al., 2005; Bosch-Bouju et al., 2013, 2014; Lobb et al., 2013).

\section{Reach-related activity}

Neural activity during reaches by the contralateral paw was recorded from the motor cortex of the hemisphere in which 6-OHDA lesion or sham surgery was performed. Neurons in both groups responded during the reach, with either firing rate increases (Fig. 4A), decreases (Fig. 4B), or combinations (Fig. 4C), which as illustrated by these examples occurred at different times during the reach, and may relate to activation of different muscle groups at different stages of the reach. We first characterized neural activity associated with reaches by the contralateral paw in control animals. A total of 68 neurons were available for this analysis, which is less than the total number of broad spike neurons recorded because some were ruled out due to motion artifact during reaching.

To visualize the timing of firing rate modulations relative to known patterns of muscle activity in the reaching task, we calculated $z$-score PETHs for all responsive neurons. These are shown for neurons from control rats that had significant increases or 

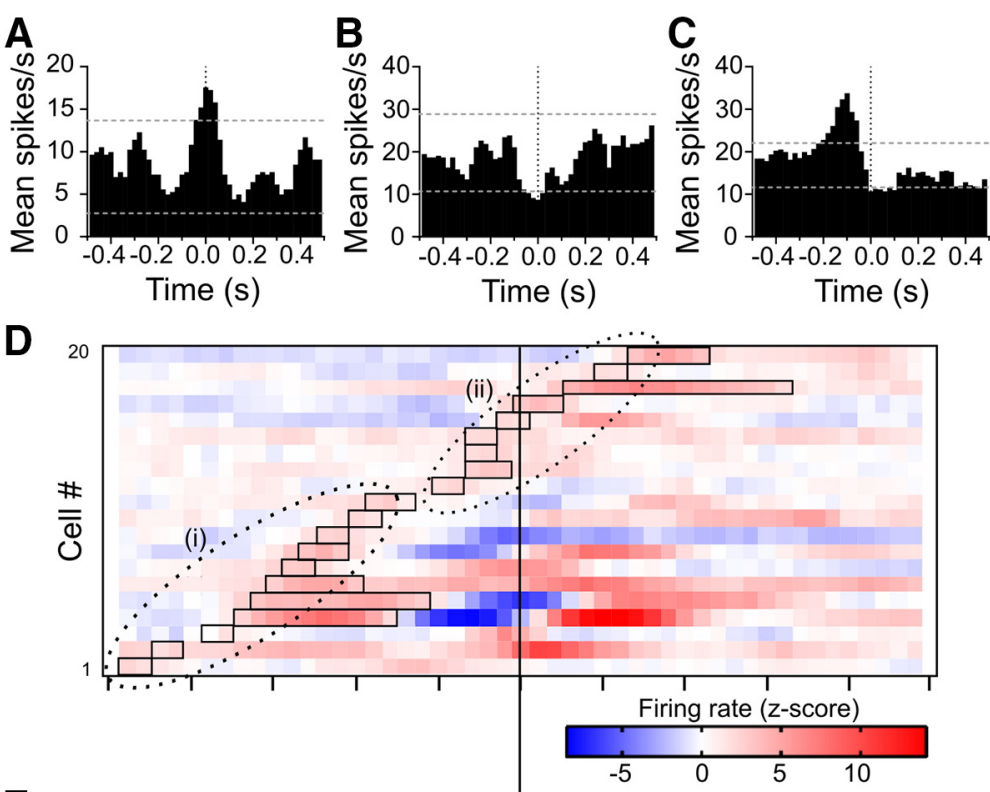

$\mathbf{E}$

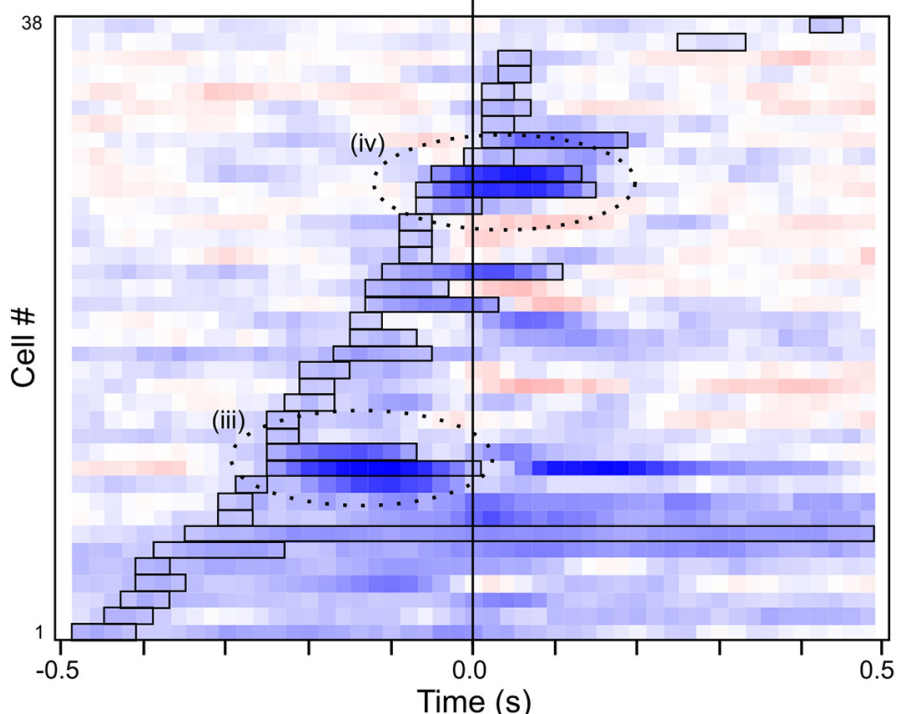

$\mathbf{F}$

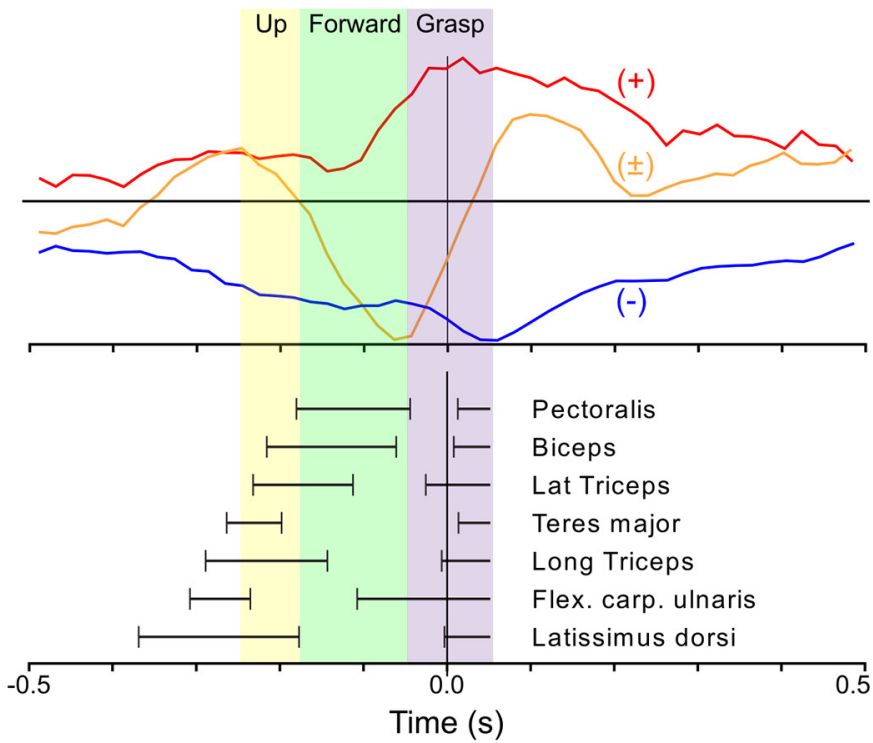

Figure 4. Characteristics of reach-related activity. $\boldsymbol{A}$, Example of individual motor cortex neuron that showed an increase in firing rate during reaches by the contralateral paw, recorded in a control rat. $\mathrm{PETH}$ are aligned (time 0 ) to end of the extension phase of the reach and onset of food grasping. Bin width $20 \mathrm{~ms}$, smoothed with three-bin rolling average. Dotted horizontal lines show decreases in firing rate in Figure 4, D and $E$, respectively. Neurons with biphasic responses were allocated to one or other display depending on the sign of first significant modulation. In cells with increases in rate (Fig. $4 D$ ) we identified two main groups of responses. The earliest (Group i) had onset latencies of the initial phasic component ranging from $\approx-0.5$ to $-0.2 \mathrm{~s}$, and this component had finished by $\approx-0.1 \mathrm{~s}$. In several of these responses there was then a rate-decrease phase, often followed by another increase. The remaining neurons (Group ii) had later onsets beginning at $\approx-0.1 \mathrm{~s}$ that spanned time 0 and were typically simple, monophasic rate increases. For neurons in which the first significant response was a rate-decrease (Fig. $4 E$ ), onset times appeared more evenly distributed throughout the reach; however there were two groups with particularly intense firing rate-decreases, one beginning at $\approx-0.25$ $s$ and ending at $\approx 0$ (Group iii), and another close to time 0 (Group iv).

To summarize the relative timing of peaks and troughs in overall population activity, Figure $4 F$ shows population average PETH. Here, we separately analyzed cells showing pure increases $(n=16)$, decreases $(n=28)$ or biphasic firing rate changes $(n=14)$, and normalized each average PETH to the maximum modulation amplitude to emphasize timing relationships. For comparison of the temporal profile of these neuronal changes with known movement components and muscle activities, Figure $4 F$ also shows a

$\leftarrow$

the \pm 2 SD thresholds for detection of a modulation. $B$, Example of a rate-decrease (lesioned rat). $\boldsymbol{C}$, Example of a biphasic modulation (control rat). $D$, Heat plot raster lines show $z$-score-normalized firing rate (smoothed with 3-bin rolling average) for all neurons in control animals with a significant firing rate increase during reaching. Neurons with a first increase as part of a biphasic response are included. Data are ordered by onset time. Open rectangles mark period of first significant rate increase for each neuron, determined using original unsmoothed PETHs. Dotted ellipses demarcate qualitative groupings of responses with similar temporal characteristics. $\boldsymbol{E}$, Heat plot raster for neurons in control animals with a firing rate decrease. Dotted ellipses demarcate two groups of neurons with particularly strong responses and similar temporal characteristics. $\boldsymbol{F}$, Colored traces show control animal population average PETH, for neurons with either only a rateincrease $(+$, red), only a decrease $(-$, blue), or a biphasic response ( \pm , orange). To emphasize timing relationships traces have been scaled to the same maximal positive or negative amplitude. Colored vertical panels show approximate times of movement phases, and black horizontal lines show timing of activation in selected muscles, previously described in video and EMG recording studies using the same task. Figure is adapted from Hyland and Jordan (1997). 

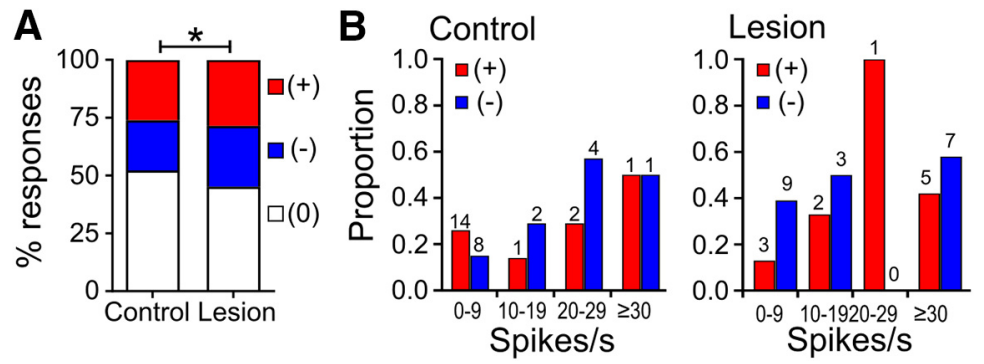

Figure 5. Comparison of proportions of motor cortex neurons responding during reaches by the contralateral paw in control and lesioned animals. $A, 0$ verall proportions. Rate increase $(+)$, red; decrease (-), blue; no change (0), white. Data for control animals calculated from PETH constructed only from the first 58 trials. ${ }^{*} p<0.05, \chi^{2}$ test. $B$, Proportions responding as a function of baseline firing rate in control (left) and lesioned animals (right). Numbers above columns show numbers of neurons in each bin.

summary of selected muscle and movement component timings previously reported during the same reach task (Whishaw and Pellis, 1990; Whishaw et al., 1992b; Hyland and Jordan, 1997).

\section{Effect of 6-OHDA lesion on reach-related activity}

From the 98 broad spike neurons recorded in lesioned rats, analysis of reach-related activity was possible for 38 obtained during sessions in which animals generated the minimum 10 reaches by the contralateral paw. Of these, more than half (26/38) responded during the reach, these responses again consisting of a mix of rate increases $(n=7)$, decreases $(n=15)$, and biphasic modulations $(n=4)$.

Before comparing responsiveness of motor cortex neurons in lesioned and control rats, we reanalyzed the control rat data presented in the previous section, using only a subset of trials performed for the PETH, so as to match the average number of trials achieved by the lesioned rats. Higher numbers of trials in the PETH increases the smoothing achieved by averaging and so could enhance the detectability of small amplitude modulations. Lesioned rats performed on average 58 reaches per recording session, whereas control rats typically performed two to three times as many. New PETHs for control rat data using only the first 58 reaches in each session yielded a smaller number (32/68) of neurons with detected responses in control animals than reported in the previous section where all available trials were used, confirming the confound risk. Therefore we used the restricted control data for all quantitative comparisons with lesioned rat data.

The comparison between control and lesioned rats for proportions of neurons showing an increase, decrease or no change in rate in the reach-end centered PETHs is shown in Figure $5 \mathrm{~A}$. For this analysis neurons with significant biphasic components were included in both the rate-increase and rate-decrease datasets. This revealed a significant difference between the groups $\left(\chi_{(2)}^{2}=8.07, p=0.017, \chi^{2}\right.$ test $)$, with overall a higher response rate in lesioned rats, due largely to an increased proportion of neurons with decreases in firing rate. Decreases may be more likely to be detected in high than low firing rate cells, and as noted above, although average firing rates were not significantly higher in lesioned animals than controls during the reaching task, there may have been a trend in that direction. We therefore analyzed the proportion of cells showing an increase or decrease in firing rate as a function of their baseline firing rate, shown in Figure $5 B$. However, in lesioned rats firing rate-decreases were the majority among the slowest firing cells as well in the fastest firing, suggesting that the dominance of inhibition was not simply secondary to differences in baseline rates.

The timing of neuronal firing rate modulations in the two Groups is illustrated in Figure 6. The heat plot displays for control animals (Fig. 6A) have fewer neurons than the overall analysis presented above, reflecting the effect of restricting the number of trials used to generate the PETH. Nevertheless, for rate-increase neurons two clusters reflecting the early and late rate-increase Groups i and ii can still be discerned. For rate-increase neurons in lesioned rats (Fig. 6B, top) a group of neurons with response onset times early during the analysis period, temporally aligned with the Group i cluster seen in control animals, is also apparent. However, in contrast to control data there was a marked paucity of rate-increase neurons with first onsets around time 0 in lesioned rats. Instead, there is a cluster of four neurons, recorded in three different rats, with responses that began somewhat earlier, at a time that such activity was not prominent in control animals. Indeed, this cluster was also differentiated by being entirely made up of biphasic response neurons, of which only one occurred in control rats in this dataset (and which had a much earlier onset). Despite these apparent differences in the timing of specific clusters of responses, with the numbers available there was no difference between Groups in the overall average response onset time $(-80 \pm 60 \mathrm{~ms}$ for lesioned rats, $-60 \pm 70 \mathrm{~ms}$ for control rats; $\left.t_{(27)}=0.182, p=0.857\right)$. However, the duration of these responses in lesioned animals $(0.07 \pm 0.03 \mathrm{~s})$ was significantly longer than in controls $\left(0.04 \pm 0.01 \mathrm{~s} ; t_{(22)}=4.022, p<0.001\right)$.

For neurons with rate-decreases there were no obvious differences in the distribution of response onset times between control (Fig. 6A, bottom) and lesioned groups (Fig. 6B, bottom), and there was no difference in the overall mean onset times (lesion $-200 \pm 180 \mathrm{~ms}$, control $-100 \pm 220 \mathrm{~ms} ; t_{(27)}=1.440, p=0.161$, unpaired $t$ test). For these responses there was also no difference in the response duration (lesioned $0.10 \pm 0.10 \mathrm{~s}$, control animals $0.10 \pm 0.11 \mathrm{~s} ; t_{(27)}=0.209, p=0.836$, unpaired $t$ test $)$.

The average activity of cortical neurons over the reach time course is shown in the population average $z$-score normalized PETH in Figure $6 C-E$. To quantify differences between the groups at different known phases of the movement, we calculated the net area under the curve (AUC) for the $z$-score PETH of each neuron, in each of six subperiods corresponding to different functional phases of the reach (see Materials and Methods). We used the same periods for both lesioned and control animals because, as quantified above (Fig. 1D), there was no difference between the groups in the durations of reaches by the contralateral paw. To avoid potential distortion of the averages by biphasic cases which could have either a first increase or decrease, we kept these neurons separate for this analysis

Figure $6 C$ shows the grand average PETH for pure rate increases. There was one neuron in a lesioned rat with an extremely strong secondary rate increase, following a much earlier significant rate-increase; the inset graph shows the data with this outlier removed. For pure rate increases, there were no significant dif- 

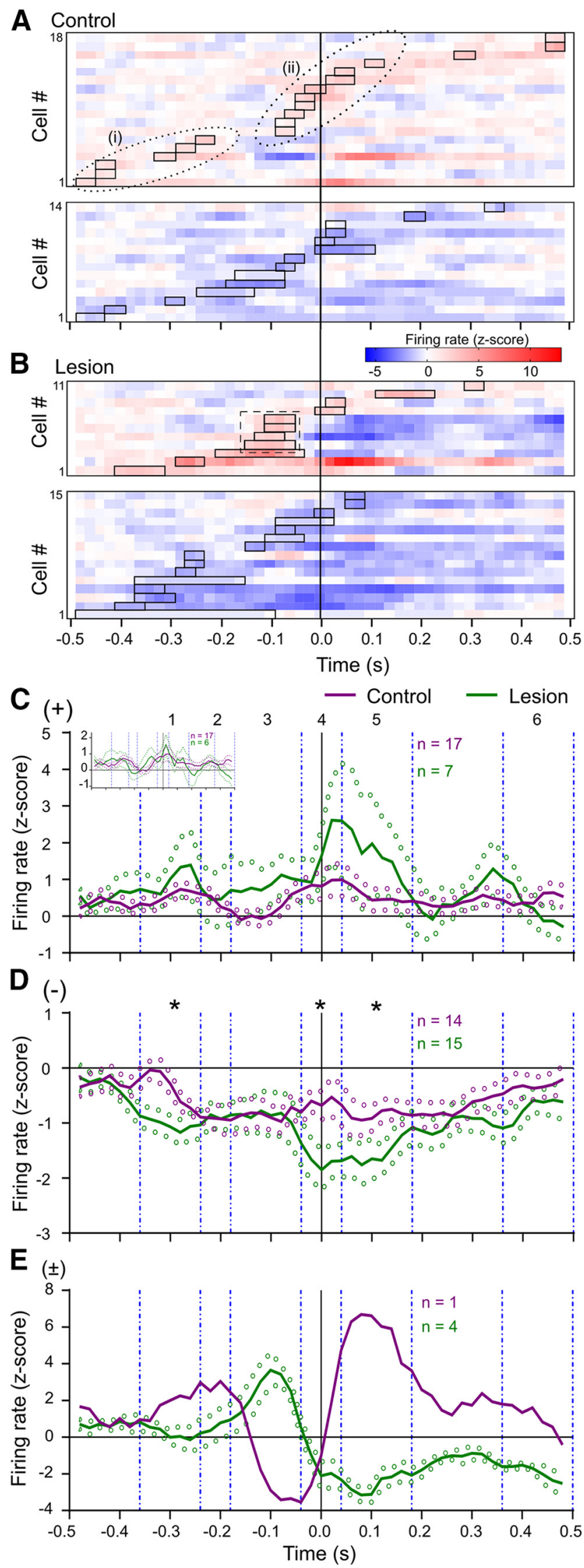

Figure 6. Effect of lesion on reach-related neural activity timing and amplitude. $\boldsymbol{A}$, Heat plots showing z-score normalized PETH from neurons in control rats, using only first 58 trials. Top, Rate-increase cells. Bottom panel, Rate-decrease cells. Other conventions are as for ferences in AUC in any epoch, with or without the outlier included. It is important to note that this analysis and the displayed average did not include the 4 neurons that formed a unique cluster in lesioned rats with onsets from $\approx-150$ to $-100 \mathrm{~ms}$, because these were all biphasic type. The temporal profile of this population average PETH therefore does not necessarily match what might have been expected from the heat plot display, where all rate increases, including those in biphasic responses were included.

Visual comparison of the color-coded $z$-score heat plots for rate decreases (Fig. $6 A, B$, bottom) suggests that the amplitude of rate-decrease responses may be greater in lesioned animals. This was confirmed by the population average analysis shown in Figure $6 D$, for several different temporal periods. In lesioned animals, there was first a significantly larger (more negative) AUC during period $\# 1$, early in the response profile (lesioned rats $-0.13 \pm 0.09$, control rats $-0.04 \pm 0.06$; $p=0.010$, MannWhitney $U$ test). Later during the reach, lesioned animals again showed more negative AUC, during both periods \#4 (lesion $-0.14 \pm 0.11$, control $-0.05 \pm 0.07 ; p<0.05$ ), and \#5 (lesion $-0.18 \pm 0.14$, control $-0.10 \pm 0.09 ; p<0.05)$.

Figure $6 E$ shows the population average for biphasic responses in lesioned rats, and the trace for the one neuron in control animals with a biphasic response. Although no statistical comparison between control and lesioned groups AUC was possible, the population average for biphasic responses in lesioned animals reflected a consistent and distinctive temporal pattern. These neurons showed an initial increase in firing rate during period \#3, followed by reduced firing rate. In contrast, the biphasic responses in the one neuron detected in the restricted data in control animals, and the population average from control animals using all available trials (Fig. $4 F$ ), had a much earlier initial peak, and a prominent trough in firing rate that temporally coincided with the peak activation of biphasic neurons in lesioned animals.

\section{Discussion}

\section{Reach-related neuronal activity}

In control rats, neural activity modulations occurred over a range of times relative to the end of the reach, similar to previous reports (Hyland, 1998; Parr-Brownlie and Hyland, 2005). Such motor cortex neuron activity is important for regulating brainstem and spinal circuits that ultimately determine muscle activity to control movement. In control animals we identified a cluster of neurons with rate increases (Group i) occurring early, before reach end. The timing of activity in these neurons, including those with bi- or triphasic modulation, was appropriately timed to be associated with triggering the recruitment of muscles highly correlated with the motion vectors involved in lift, aim and advance components of the reach (Whishaw and Pellis, 1990; Whishaw et al., 1992b; Hyland and Jordan, 1997).

$\leftarrow$

Figure $4, D$ and $E$. The dotted ovals span the same time ranges as in Figure 4. B, Heat plots for neurons in lesioned animals. Dashed line box highlights a group of rate-increase neurons with peak activity as part of a biphasic responses, not seen in controls. C, Traces show population average z-scored firing rate \pm SEM (solid and dotted lines, respectively), for neurons classified as having pure rate increases in control (purple) and lesioned (green) animals. Data were smoothed with a three-bin rolling average. Vertical dashed lines demarcate subperiods 1-6 used for statistical comparisons. Inset graph shows data after excluding one outlier neuron with a very large amplitude response from the lesioned group. $\boldsymbol{D}$, As for $\boldsymbol{C}$, for neurons with pure rate-decreases. ${ }^{*} p<0.05$, Mann-Whitney $U$ test comparing average area under the curve in each subperiod. $\boldsymbol{E}$, As for $\boldsymbol{D}$, for neurons with biphasic responses. Only one neuron in control animals had a significant biphasic response component in PETH with reduced trial number, so no statistical contrasts were possible. 
A second cluster of neurons (Group ii) in control animals had simple rate increases at the end of the advance component of the reach, appropriately timed to be involved in controlling distal movements occurring at this phase of the reach. At this time, rats extend the digits as the paw decelerates, elbow and wrist movements combine to pronate the paw over the food in an "arpeggio" pattern, and then grasping movements occur, involving independent control of different groups of digits (Whishaw et al., 1994). Unlike for proximal muscles, direct comparison of the neural activity we recorded with activity in muscles involved in these distal components in rat is not possible, because EMG data are lacking. However, in support, primates show specific grasp related activity in distal muscles at equivalent phases of reach-tograsp movements, and specific populations of primate motor cortex neurons show activity correlations with such distal movements (e.g., Brochier et al., 2004; Umiltà et al., 2007; VargasIrwin et al., 2010).

\section{Effect of 6-OHDA lesion on reach-related neuronal activity}

A main finding of the present study was that rats with unilateral dopamine lesion showed several differences in contralateral motor cortex activity compared with that seen in control animals, when they attempted to use their affected paw to reach and grasp food. This was despite the fact that reaches that were performed by this limb in lesioned rats did not differ in velocity from those performed by control rats. First, there was a significant increase in the proportion of modulated neurons, due in particular to an increase in the proportion with rate-decreases. In addition, the population average analysis for these latter neurons revealed a significantly greater level of decrease in lesioned animals than in control animals during particular phases of the reach. These findings suggest a shift in the balance of net excitation and inhibition for different populations of cortical neurons in the lesioned animals. Second, whereas in this dataset, we did not detect any significant change in population average amplitudes for rateincrease responses, there was a significant increase in the duration of these responses in lesioned rats. Such an effect could reflect generally less temporally focused activity in the excitatory and inhibitory input pathways, that determine the timing of net increases; or a specific loss of activity more tightly coupled to the reach-event used to center the PETH, which in this case was the moment in the reach where the paw approached the food target and preparations for, and grasping itself would be occurring.

Previous studies in animal models and human Parkinson's disease have found a range of deficits in motor cortex activation. In rats, we previously found reduced motor cortex reach-related rate increases in an acute drug-induced model of parkinsonism (Parr-Brownlie and Hyland, 2005). This difference may relate to acute versus chronic nature of the models. Primate studies in chronic lesion models have focused on simple, single-joint extension-flexion tasks without distal fine motor control elements but have reported various deficits including loss of neurons with reciprocal activity (Doudet et al., 1990), reduced incidence and amplitude of responses (Watts and Mandir, 1992), and reduction in the extent to which neural activity encodes movement parameters (Pasquereau et al., 2016). In studies of regional blood flow in human Parkinson's disease there are conflicting data, including both decreases (75\% of studies) and increases in movementrelated modulation (reviewed by Herz et al., 2014). One possible mechanism underpinning such changes in movement-related neuronal activity is altered responsiveness of neurons to inputs, subsequent to loss of cortical dopamine (Guo et al., 2015). Such an effect on excitability could for instance account for the fact that dopamine depletion reduces the motor cortex area mapped to distal muscles in rat, as defined using intracortical microstimulation in anesthetized animals (Viaro et al., 2011). However, in conscious animals changes are often specific to different phases of behavior, thus involving a temporal aspect that would more likely in turn reflect changes in the temporal dynamics of activity in input pathways.

Indeed, shifts in the patterning and timing of activity relative to temporally specific components of the reach was the third main class of finding in the present study. Similar to controls, in lesioned animals there was a population of neurons that showed early "Group i" type activity temporally associated with reach onset, when proximal muscles are active to lift and propel the limb. That such neuronal activity is present is not surprising, given that a reach, with successful penetration by the paw into the feeder, had to occur in order for the analysis to be performed. However, even at this time there was a significant change in the amplitude of rate-decrease responses. Such subtle changes may relate to the fact that while unilaterally lesioned animals are able on occasion propel the affected limb toward the feeder, there are nevertheless deficits in the organization of these early reach components (Miklyaeva et al., 1994).

In contrast to the retention of some activity matching Group i, in lesioned rats there was impoverished expression of the Group ii class of neurons defined in control animals, i.e., those with rate-increases beginning later in the reach and spanning the reach-end period. Further, a new class of cells with biphasic responses became prominent. These had a particular temporal pattern with an initial rate-increase peak occurring shortly before reach-end, followed by sustained rate decrease. This was quite unlike and almost a mirror image of the pattern in control animals, in which the first peak was much earlier in the reach, followed by a trough-peak sequence. Together with the significantly enhanced amplitude of rate-decreases in lesioned animals described above, these data point to quite differently organized cortical activity in unilateral 6-OHDA lesioned animals during the end stages of the reach, when fine distal movements associated with shaping of the paw and digits for successful food grasping are normally made by intact rats (Whishaw and Pellis, 1990; Whishaw et al., 1992b).

Such temporally specific changes are likely to reflect changes in the dynamics of activity in afferent pathways. Indeed, we previously found the same time period to be a main focus of changes in the motor thalamus in unilateral 6-OHDA lesioned rats performing the same task (Bosch-Bouju et al., 2014). These changes included alterations in both the organization of reach-related overall average firing rate, and in the timing of thalamic low threshold calcium spike-like bursts, and presumably in turn reflect upstream changes in basal ganglia processing that occur following dopamine depletion (Bosch-Bouju et al., 2013, 2014).

The findings of particular deficits in neural activity associated with the end phase of the reach when grasping movements are made is consistent with and probably account for the marked deficits in fine control of grasping that occur in rat models of Parkinson's disease. These deficits have been well characterized in both spontaneous food handling (Whishaw et al., 1997b) and reach-to-grasp tasks (Miklyaeva et al., 1994; Whishaw et al., 1997a). Specifically, in the same reach-to-grasp task used here rats with unilateral 6-OHDA lesions lack the refined combination of timed digit extension and paw pronation, failing to generate the arpeggio pattern to position the paw over food in preparation for grasp, and poor grasping, with whole-paw digit closure instead of the partly independent digit movements seen in control rats (Mikly- 
aeva et al., 1994). As reviewed by Whishaw et al. (2002), and reiterated in subsequent studies (e.g., Schettino et al., 2004; Ansuini et al., 2010; Lukos et al., 2014; Parma et al., 2014), patients with Parkinson's disease also have significant problems with manual dexterity, and particular difficulties in coordinating the complex interplay of proximal and distal movement components required to achieve properly timed grasping. Such movements are critical to the practical usefulness of the upper limb, yet are particularly resistant to improvement by Parkinson's disease therapies (Melvin et al., 2005). Together, these data highlight the need to continue developing therapeutic interventions acting at the cortical level (Dileone et al., 2017; Yang et al., 2018), that are particularly targeted to improving recruitment of upper motor neurons within the representations of the distal musculature.

\section{References}

Albin RL, Young AB, Penney JB (1989) The functional anatomy of basal ganglia disorders. Trends Neurosci 12:366-375.

Ansuini C, Begliomini C, Ferrari T, Castiello U (2010) Testing the effects of end-goal during reach-to-grasp movements in Parkinson's disease. Brain Cogn 74:169-177.

Barthó P, Hirase H, Monconduit L, Zugaro M, Harris KD, Buzsáki G (2004) Characterization of neocortical principal cells and interneurons by network interactions and extracellular features. J Neurophysiol 92:600-608.

Benecke R, Rothwell JC, Dick JP, Day BL, Marsden CD (1987) Simple and complex movements off and on treatment in patients with Parkinson's disease. J Neurol Neurosurg Psychiatry 50:296-303.

Boraud T, Bezard E, Bioulac B, Gross CE (2002) From single extracellular unit recording in experimental and human parkinsonism to the development of a functional concept of the role played by the basal ganglia in motor control. Prog Neurobiol 66:265-283.

Bosch-Bouju C, Hyland BI, Parr-Brownlie LC (2013) Motor thalamus integration of cortical, cerebellar and basal ganglia information: implications for normal and parkinsonian conditions. Front Comput Neurosci 7:163.

Bosch-Bouju C, Smither RA, Hyland BI, Parr-Brownlie LC (2014) Reduced reach-related modulation of motor thalamus neural activity in a rat model of Parkinson's disease. J Neurosci 34:15836-15850.

Brochier T, Umiltà MA (2007) Cortical control of grasp in non-human primates. Curr Opin Neurobiol 17:637-643.

Brochier T, Spinks RL, Umiltà MA, Lemon RN (2004) Patterns of muscle activity underlying object-specific grasp by the macaque monkey. J Neurophysiol 92:1770-1782.

Castiello U, Bennett KM (1994) Parkinson's disease: reorganization of the reach to grasp movement in response to perturbation of the distal motor patterning. Neuropsychologia 32:1367-1382.

Costa RM, Lin SC, Sotnikova TD, Cyr M, Gainetdinov RR, Caron MG, Nicolelis MA (2006) Rapid alterations in corticostriatal ensemble coordination during acute dopamine-dependent motor dysfunction. Neuron 52:359-369.

Debeir T, Ginestet L, François C, Laurens S, Martel JC, Chopin P, Marien M, Colpaert F, Raisman-Vozari R (2005) Effect of intrastriatal 6-OHDA lesion on dopaminergic innervation of the rat cortex and globus pallidus. Exp Neurol 193:444-454.

Degos B, Deniau JM, Chavez M, Maurice N (2013) Subthalamic nucleus high-frequency stimulation restores altered electrophysiological properties of cortical neurons in parkinsonian rat. PLoS One 8:e83608.

Dejean C, Nadjar A, Le Moine C, Bioulac B, Gross CE, Boraud T (2012) Evolution of the dynamic properties of the cortex-basal ganglia network after dopaminergic depletion in rats. Neurobiol Dis 46:402-413.

Dileone M, Carrasco-López MC, Segundo-Rodriguez JC, Mordillo-Mateos L, López-Ariztegui N, Alonso-Frech F, Catalan-Alonso MJ, Obeso JA, Oliviero A, Foffani G (2017) Dopamine-dependent changes of cortical excitability induced by transcranial static magnetic field stimulation in Parkinson's disease. Sci Rep 7:4329.

Doudet DJ, Gross C, Arluison M, Bioulac B (1990) Modifications of precentral cortex discharge and EMG activity in monkeys with MPTP-induced lesions of DA nigral neurons. Exp Brain Res 80:177-188.

Ferguson JE, Boldt C, Redish AD (2009) Creating low-impedance tetrodes by electroplating with additives. Sens Actuators A Phys 156:388-393.

Galvan A, Devergnas A, Wichmann T (2015) Alterations in neuronal activ- ity in basal ganglia-thalamocortical circuits in the parkinsonian state. Front Neuroanat 9:5.

Galvan A, Hu X, Smith Y, Wichmann T (2016) Effects of optogenetic activation of corticothalamic terminals in the motor thalamus of awake monkeys. J Neurosci 36:3519-3530.

Gaspar P, Duyckaerts C, Alvarez C, Javoy-Agid F, Berger B (1991) Alterations of dopaminergic and noradrenergic innervations in motor cortex in Parkinson's disease. Ann Neurol 30:365-374.

Goldberg JA, Boraud T, Maraton S, Haber SN, Vaadia E, Bergman H (2002) Enhanced synchrony among primary motor cortex neurons in the 1-methyl-4-phenyl-1,2,3,6-tetrahydropyridine primate model of Parkinson's disease. J Neurosci 22:4639-4653.

Guo L, Xiong H, Kim JI, Wu YW, Lalchandani RR, Cui Y, Shu Y, Xu T, Ding JB (2015) Dynamic rewiring of neural circuits in the motor cortex in mouse models of Parkinson's disease. Nat Neurosci 18:1299-1309.

Herz DM, Eickhoff SB, Løkkegaard A, Siebner HR (2014) Functional neuroimaging of motor control in Parkinson's disease: a meta-analysis. Hum Brain Mapp 35:3227-3237.

Hosp JA, Pekanovic A, Rioult-Pedotti MS, Luft AR (2011) Dopaminergic projections from midbrain to primary motor cortex mediate motor skill learning. J Neurosci 31:2481-2487.

Hyland B (1998) Neural activity related to reaching and grasping in rostral and caudal regions of rat motor cortex. Behav Brain Res 94:255-269.

Hyland BI, Jordan VM (1997) Muscle activity during forelimb reaching movements in rats. Behav Brain Res 85:175-186.

Jarratt H, Hyland B (1999) Neuronal activity in the rat red nucleus during forelimb reach-to-grasp movements. Neuroscience 88:629-642.

Kaneoke Y, Vitek JL (1996) Burst and oscillation as disparate neuronal properties. J Neurosci Methods 68:211-223.

Klein A, Sacrey LA, Whishaw IQ, Dunnett SB (2012) The use of rodent skilled reaching as a translational model for investigating brain damage and disease. Neurosci Biobehav Rev 36:1030-1042.

Kolb B, Whishaw IQ (1983) Dissociation of the contributions of the prefrontal, motor, and parietal cortex to the control of movement in the rat: an experimental review. Can J Psychol 37:211-232.

Latash ML, Anson JG (1996) What are abnormal movements in atypical populations? Behav Brain Sci 19:55-106.

Lewis BL, O’Donnell P (2000) Ventral tegmental area afferents to the prefrontal cortex maintain membrane potential 'up' states in pyramidal neurons via $\mathrm{D}(1)$ dopamine receptors. Cereb Cortex 10:1168-1175.

Lindenbach D, Conti MM, Ostock CY, Dupre KB, Bishop C (2015) Alterations in primary motor cortex neurotransmission and gene expression in hemi-parkinsonian rats with drug-induced dyskinesia. Neuroscience 310: $12-26$.

Lobb CJ, Zaheer AK, Smith Y, Jaeger D (2013) In vivo electrophysiology of nigral and thalamic neurons in alpha-synuclein-overexpressing mice highlights differences from toxin-based models of parkinsonism. J Neurophysiol 110:2792-2805.

Lukos JR, Poizner H, Sage JI (2014) Hand function in Parkinson's disease. In: Hand function: a practical guide to assessment (Duruöz MT, ed), pp 133-149. New York: Springer.

Melvin KG, Doan J, Pellis SM, Brown L, Whishaw IQ, Suchowersky O (2005) Pallidal deep brain stimulation and L-dopa do not improve qualitative aspects of skilled reaching in Parkinson's disease. Behav Brain Res 160: $188-194$.

Miklyaeva EI, Castañeda E, Whishaw IQ (1994) Skilled reaching deficits in unilateral dopamine-depleted rats: impairments in movement and posture and compensatory adjustments. J Neurosci 14:7148-7158.

Olsson M, Nikkhah G, Bentlage C, Björklund A (1995) Forelimb akinesia in the rat parkinson model: differential effects of dopamine agonists and nigral transplants as assessed by a new stepping test. J Neurosci 15 : 3863-3875.

Parma V, Zanatto D, Straulino E, Scaravilli T, Castiello U (2014) Kinematics of the reach-to-grasp movement in vascular parkinsonism: a comparison with idiopathic Parkinson's disease patients. Front Neurol 5:75.

Parr-Brownlie LC, Hyland BI (2005) Bradykinesia induced by dopamine D2 receptor blockade is associated with reduced motor cortex activity in the rat. J Neurosci 25:5700-5709.

Parr-Brownlie LC, Poloskey SL, Flanagan KK, Eisenhofer G, Bergstrom DA, Walters JR (2007) Dopamine lesion-induced changes in subthalamic nucleus activity are not associated with alterations in firing rate or pattern 
in layer $\mathrm{V}$ neurons of the anterior cingulate cortex in anesthetized rats. Eur J Neurosci 26:1925-1939.

Parr-Brownlie LC, Poloskey SL, Bergstrom DA, Walters JR (2009) Parafascicular thalamic nucleus activity in a rat model of Parkinson's disease. Exp Neurol 217:269-281.

Pasquereau B, Turner RS (2011) Primary motor cortex of the parkinsonian monkey: differential effects on the spontaneous activity of pyramidal tract-type neurons. Cereb Cortex 21:1362-1378.

Pasquereau B, Turner RS (2013) Primary motor cortex of the parkinsonian monkey: altered neuronal responses to muscle stretch. Front Syst Neurosci 7:98.

Pasquereau B, DeLong MR, Turner RS (2016) Primary motor cortex of the parkinsonian monkey: altered encoding of active movement. Brain 139:127-143.

Paxinos G, Watson C (2007) The rat brain in stereotaxic coordinates, Ed 6. Amsterdam: Elsevier.

Pessiglione M, Guehl D, Rolland AS, François C, Hirsch EC, Féger J, Tremblay L (2005) Thalamic neuronal activity in dopamine-depleted primates: evidence for a loss of functional segregation within basal ganglia circuits. J Neurosci 25:1523-1531.

Planetta PJ, McFarland NR, Okun MS, Vaillancourt DE (2014) MRI reveals brain abnormalities in drug-naive Parkinson's disease. Exerc Sport Sci Rev 42:12-22.

Sacrey LA, Alaverdashvili M, Whishaw IQ (2009) Similar hand shaping in reaching-for-food (skilled reaching) in rats and humans provides evidence of homology in release, collection, and manipulation movements. Behav Brain Res 204:153-161.

Schettino LF, Rajaraman V, Jack D, Adamovich SV, Sage J, Poizner H (2004) Deficits in the evolution of hand preshaping in Parkinson's disease. Neuropsychologia 42:82-94.

Seamans JK, Gorelova N, Durstewitz D, Yang CR (2001a) Bidirectional dopamine modulation of GABAergic inhibition in prefrontal cortical pyramidal neurons. J Neurosci 21:3628-3638.

Seamans JK, Durstewitz D, Christie BR, Stevens CF, Sejnowski TJ (2001b) Dopamine D1/D5 receptor modulation of excitatory synaptic inputs to layer V prefrontal cortex neurons. Proc Natl Acad Sci U S A 98:301-306.

Seeger-Armbruster S, Bosch-Bouju C, Little ST, Smither RA, Hughes SM, Hyland BI, Parr-Brownlie LC (2015) Patterned, but not tonic, optogenetic stimulation in motor thalamus improves reaching in acute druginduced parkinsonian rats. J Neurosci 35:1211-1216.

Thabit MN, Nakatsuka M, Koganemaru S, Fawi G, Fukuyama H, Mima T (2011) Momentary reward induce changes in excitability of primary motor cortex. Clin Neurophysiol 122:1764-1770.

Umiltà MA, Brochier T, Spinks RL, Lemon RN (2007) Simultaneous recording of macaque premotor and primary motor cortex neuronal populations reveals different functional contributions to visuomotor grasp. J Neurophysiol 98:488-501.

Vargas-Irwin CE, Shakhnarovich G, Yadollahpour P, Mislow JM, Black MJ, Donoghue JP (2010) Decoding complete reach and grasp actions from local primary motor cortex populations. J Neurosci 30:9659-9669.

Vergara-Aragon P, Gonzalez CL, Whishaw IQ (2003) A novel skilledreaching impairment in paw supination on the "good" side of the hemiparkinson rat improved with rehabilitation. J Neurosci 23:579-586.

Viaro R, Morari M, Franchi G (2011) Progressive motor cortex functional reorganization following 6-hydroxydopamine lesioning in rats. J Neurosci 31:4544-4554.
Walters JR, Hu D, Itoga CA, Parr-Brownlie LC, Bergstrom DA (2007) Phase relationships support a role for coordinated activity in the indirect pathway in organizing slow oscillations in basal ganglia output after loss of dopamine. Neuroscience 144:762-776.

Watts RL, Mandir AS (1992) The role of motor cortex in the pathophysiology of voluntary movement deficits associated with parkinsonism. Neurologic Clinics 10:451-469.

Whishaw IQ (2000) Loss of the innate cortical engram for action patterns used in skilled reaching and the development of behavioral compensation following motor cortex lesions in the rat. Neuropharmacology 39:788-805.

Whishaw IQ, Coles BL (1996) Varieties of paw and digit movement during spontaneous food handling in rats: postures, bimanual coordination, preferences, and the effect of forelimb cortex lesions. Behav Brain Res 77:135-148.

Whishaw IQ, Gorny B (1994) Arpeggio and fractionated digit movements used in prehension by rats. Behav Brain Res 60:15-24.

Whishaw IQ, Pellis SM (1990) The structure of skilled forelimb reaching in the rat: a proximally driven movement with a single distal rotatory component. Behav Brain Res 41:49-59.

Whishaw IQ, Dringenberg HC, Pellis SM (1992a) Spontaneous forelimb grasping in free feeding by rats: motor cortex aids limb and digit positioning. Behav Brain Res 48:113-125.

Whishaw IQ, Pellis SM, Gorny BP (1992b) Skilled reaching in rats and humans: evidence for parallel development or homology. Behav Brain Res 47:59-70.

Whishaw IQ, Gorny B, Tran-Nguyen LT, Castañeda E, Miklyaeva EI, Pellis SM (1994) Making two movements at once: impairments of movement, posture, and their integration underlie the adult skilled reaching deficit of neonatally dopamine-depleted rats. Behav Brain Res 61:65-77.

Whishaw IQ, Woodward NC, Miklyaeva E, Pellis SM (1997a) Analysis of limb use by control rats and unilateral DA-depleted rats in the montoya staircase test: movements, impairments and compensatory strategies. Behav Brain Res 89:167-177.

Whishaw IQ, Coles BL, Pellis SM, Miklyaeva EI (1997b) Impairments and compensation in mouth and limb use in free feeding after unilateral dopamine depletions in a rat analog of human Parkinson's disease. Behav Brain Res 84:167-177.

Whishaw IQ, Suchowersky O, Davis L, Sarna J, Metz GA, Pellis SM (2002) Impairment of pronation, supination, and body co-ordination in reachto-grasp tasks in human Parkinson's disease (PD) reveals homology to deficits in animal models. Behav Brain Res 133:165-176.

Wichmann T, Dostrovsky JO (2011) Pathological basal ganglia activity in movement disorders. Neuroscience 198:232-244.

Wilson FA, O'Scalaidhe SP, Goldman-Rakic PS (1994) Functional synergism between putative gamma-aminobutyrate-containing neurons and pyramidal neurons in prefrontal cortex. Proc Natl Acad Sci U S A 91:4009-4013.

Wu T, Hallett M (2013) Functional MRI in idiopathic Parkinson disease and parkinsonism. In: Neuroimaging of movement disorders (Nahab FB, Hattori N, eds), pp 143-157. Totowa, NJ: Humana.

Yang C, Guo Z, Peng H, Xing G, Chen H, McClure MA, He B, He L, Du F, Xiong L, Mu Q (2018) Repetitive transcranial magnetic stimulation therapy for motor recovery in Parkinson's disease: a meta-analysis. Brain Behav 8:e01132. 